\title{
La configuración de las condiciones laborales de la enfermería en el Área Metropolitana de Buenos Aires: un análisis en el cruce del orden de género y la organización del sistema de salud
}

\author{
The configuration of nursing labor conditions in the \\ Buenos Aires Metropolitan Area: An analysis at the \\ intersection of the gender order and the organization \\ of the health system
}

Francisca Pereyra ${ }^{1}$, Ariela Micha ${ }^{2}$

'Licenciada en Sociología, Doctora en Sociología. Investigadora-Docente, Universidad Nacional de General Sarmiento, Buenos Aires, Argentina. $\square$ (iD)

'Licenciada en Economía, Magíster en Estudios de Desarrollo. Becaria doctoral del Consejo Nacional de Investigaciones Científicas y Técnicas (CONICET), Universidad Nacional de General Sarmiento, Buenos Aires, Argentina. $\square$ (iD
RESUMEN El artículo indaga dos factores centrales que contribuyen a la configuración del endeble panorama laboral que presenta la enfermería en Argentina. El primer objetivo se centra en explorar cómo incide en las condiciones de trabajo el componente de cuidado, culturalmente asociado a imágenes sobre saberes y habilidades supuestamente "innatas" de las mujeres y, el segundo, en indagar cómo la forma en que se organiza la provisión de los servicios de salud en nuestro país intensifica la vulnerabilidad de esta ocupación. En cuanto a la metodología, el trabajo de campo realizado en el Área Metropolitana de Buenos Aires incluyó entrevistas en profundidad a referentes de la actividad, así como entrevistas grupales con enfermeras y enfermeros. Entre los resultados se muestra, por un lado, cómo la devaluación social del cuidado está presente en discursos de quienes ejercen la ocupación, así como en las prácticas y políticas institucionales del sector salud. Por otro lado, se evidencia cómo la descentralización y la fragmentación del sistema de salud se presentan como obstáculos adicionales, dificultando la articulación de reclamos laborales.

PALABRAS CLAVES Personal de Enfermería; Condiciones de Trabajo; Trabajo de Mujeres; Argentina.

\begin{abstract}
The article explores two key factors which contribute to shape the poor working conditions of nursing in Argentina. A first objective focuses on exploring the effect of the occupation's care component, closely associated with cultural images of "inherent" female qualities, on working conditions. A second objective aims to examine the way in which the organization of health services provision in Argentina intensifies the vulnerability of this occupation. Regarding the methodology, the fieldwork conducted in the Metropolitan Area of Buenos Aires included in-depth interviews with key informants and group interviews with nurses. Among the results, on the one hand it is shown how the social devaluation of care is reflected in the discourse of those who perform the occupation as well as in institutional practices and policies in the health sector. On the other hand, it is shown that the decentralization and fragmentation of the health system act as additional obstacles hampering the articulation of labor demands.
\end{abstract}

KEY WORDS Nursing Staff; Working Conditions; Women, Working; Argentina. 


\section{INTRODUCCIÓN}

El análisis de las condiciones laborales de las y los trabajadores del cuidado ha cobrado creciente relevancia en la agenda feminista de los últimos años. La preocupación se inscribe dentro de una línea de trabajo relativamente reciente que proviene de la economía feminista anglosajona -pero con importantes desarrollos a nivel regional- que ha enfatizado la categoría de cuidado más que otras precedentes tales como el trabajo doméstico o trabajo reproductivo ${ }^{(1,2,3,4)}$. Mientras que los trabajos pioneros del feminismo se ocuparon de poner en evidencia la falta de valoración económica del trabajo reproductivo que realizan las mujeres de forma no remunerada al interior del hogar, resaltando que supone una contribución fundamental a la reproducción de la fuerza de trabajo ${ }^{(5,6,7)}$, los estudios basados en la categoría de cuidado buscan desplazar la atención del lugar donde se desarrollan las tareas y centrarla sobre el tipo de trabajo asignado a las mujeres y su persistente devaluación social y económica. Este enfoque permite expandir las fronteras más allá del trabajo reproductivo al interior de los hogares, para observar lo que sucede con el desarrollo de ciertas tareas que implican una extensión de habilidades supuestamente "femeninas" en la esfera remunerada del mercado.

Tendencias como el aumento de la participación laboral de las mujeres en las últimas décadas y cambios demográficos - por ejemplo, el envejecimiento de la población o la disminución del tamaño de los hogares con la consecuente disminución de redes familiares de apoyo- han generado una mayor demanda de servicios remunerados de cuidado. Este fenómeno implica una necesaria (y nueva) atención dirigida hacia el desarrollo de las ocupaciones relacionadas con el cuidado. A su vez, el importante nivel de feminización de estas ocupaciones indica que constituyen un sector que genera oportunidades de empleo para muchas mujeres ${ }^{(1)}$.

¿Quiénes son las y los trabajadores del cuidado? En general, se considera como tales a quienes desempeñan actividades que contribuyan a la salud, la seguridad física, y el desarrollo de habilidades cognitivas, físicas o emocionales de las personas, y que interactúan directamente con los receptores del servicio en cuestión ${ }^{(8)}$. Típicamente, estas ocupaciones incluyen docentes de todos los niveles, terapeutas, médicas/os y enfermeras/ os, y se concentran en el sector salud y en el de educación.

Los estudios existentes han abordado el tema desde el punto de vista de las condiciones laborales de las y los trabajadores del cuidado frente a las condiciones de trabajo de las ocupaciones no relacionadas con el cuidado, bajo la hipótesis de que, al tratarse de tareas socialmente poco valoradas -asociadas con aptitudes y habilidades supuestamente femeninas e innatas- podrían sufrir una potencial penalización en el mercado en términos de remuneraciones y condiciones de trabajo. Asimismo, se han explorado qué otras variables -fundamentalmente factores de tipo político-institucional que hacen a la forma en que se organiza, se regula y se disputa cada dinámica ocupacional particular- combinadas con el cuidado atenúan o incrementan la penalización que implica desempeñarse laboralmente en este tipo de ocupaciones $^{(8,9,10,11)}$.

Este trabajo se propone contribuir a este cúmulo de conocimientos a partir del caso específico de la enfermería en el Área Metropolitana de Buenos Aires (AMBA) ${ }^{[a]}$.

El ejercicio de la enfermería implica la prestación de un servicio social clave. El personal de enfermería, con base en sus conocimientos y habilidades, es quien proporciona los cuidados necesarios para los procesos de promoción, recuperación y mantenimiento de la salud ${ }^{(14)}$. A diferencia de otras ocupaciones del cuidado, en las que este constituye un componente entre otros, la enfermería reviste la particularidad de definirse a sí misma en términos de su objetivo y misión de brindar cuidado. Asimismo, y en línea con lo señalado para las ocupaciones del cuidado en general, la enfermería constituye una ocupación altamente feminizada ${ }^{(15)}$. 
En términos generales, la bibliografía existente sobre el tema en Argentina relata una serie de problemas "típicos" de esta ocupación que marcan un nivel significativo de precariedad: salarios bajos, pluriempleo, estrés laboral y falta de equipamiento e infraestructura adecuados, entre algunos de los

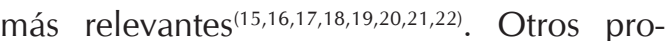
blemas frecuentemente señalados tienen que ver con la permanente exposición a riesgos biológicos, químicos y físicos, con patologías osteomusculares y articulares producidas por esfuerzos físicos que demandan las tareas, así como también alteraciones del patrón de sueño y fatiga, sobre todo entre las mujeres que trabajan en horarios nocturnos y deben compatibilizar el trabajo con las responsabilidades domésticas ${ }^{(10)}$. Asimismo, la falta de recursos humanos conforma uno de los problemas fundamentales que afectan a la ocupación, y este déficit de trabajadores se manifiesta no solo por la escasez en el número de enfermeras/os en ejercicio y la consecuente sobrecarga laboral que esto implica, sino también por su calificación, conformando un déficit cuali-cuantitativo de recursos humanos ${ }^{(23,24)}$.

El propósito final de este texto es el de contribuir al conocimiento de los factores claves que estructuran las condiciones laborales de la enfermería a fin de generar elementos que permitan pensar en políticas dirigidas a esta ocupación. En este sentido, se propone abordar dos factores que, si bien no pretenden agotar el análisis de la problemática, se presentaron como centrales en nuestra indagación sobre la configuración del panorama laboral de la enfermería.

De esta manera, el primer objetivo específico se centra en explorar cómo incide en las condiciones de trabajo el componente de cuidado. La bibliografía sobre el tema sostiene que las labores de cuidado, fuertemente asociadas con imágenes tradicionales de género $-y$, por ende, identificadas con aptitudes y habilidades supuestamente inherentes a la condición femenina- dificultan su percepción en tanto "auténtico trabajo". De esta manera, la primera parte del artículo aborda las dificultades que el cuidado implica para construir un discurso "profesionalizado" de la ocupación, así como la forma en que esta imagen devaluada del cuidado se refleja en prácticas y políticas institucionales del sector salud.

No obstante, tal como ya fue señalado, los análisis sobre el tema evidencian también que los contextos políticos-institucionales en los que se desarrollan este tipo de ocupaciones revisten suma importancia a la hora de reforzar o mitigar la devaluación social del cuidado. Por esta razón, el segundo objetivo específico es el de indagar cómo la forma en que se organiza la provisión de los servicios de salud en nuestro país intensifica la vulnerabilidad de esta ocupación. En particular, nos centraremos en torno a cómo la descentralización y la fragmentación del sector salud marcan diferencias entre las experiencias laborales y obstaculizan las posibilidades de articulación de los reclamos.

\section{METODOLOGÍA}

La metodología utilizada fue cualitativa. El trabajo de campo se desarrolló entre septiembre de 2013 y mayo de 2014 en el ámbito del AMBA. Por un lado, se realizaron una serie de entrevistas en profundidad con referentes de la ocupación, entre ellos, directoras de carreras de enfermería de universidades públicas y privadas, directoras de departamentos de enfermería (para el sector público se incluyeron referentes nacionales, provinciales y municipales) y referentes sindicales del sector. Por otro lado, se realizaron dos encuentros grupales con estudiantes avanzados de la licenciatura en Enfermería que ya estaban ejerciendo la profesión: uno en una universidad privada de la Ciudad Autónoma de Buenos Aires, en el que participaron 26 estudiantes, y el otro en una universidad pública del Gran Buenos Aires, en el que participaron 15 estudiantes. Las edades oscilaron entre los 25 y los 56 años. En ambos casos predominaron las mujeres (más del $90 \%$ del total) mayores de 40 años (más del $60 \%$ de los casos). Si bien la gran mayoría trabajaba 
directamente atendiendo pacientes, también participaron supervisoras de piso, coordinadores de área y una jefa de departamento. Las personas entrevistadas se encontraban insertas en diversos ámbitos: hospitales nacionales, provinciales y municipales; así como también clínicas, sanatorios y geriátricos privados.

En lo que respecta a los criterios éticos, se solicitó y obtuvo el consentimiento expreso de las personas entrevistadas. Al invitarlas a participar en la investigación se les brindó información acerca de la finalidad y la duración, la metodología y los criterios de finalización de su participación, enfatizando el derecho a retirarse en cualquier momento si así lo deseaban. Además, con el propósito de preservar la confidencialidad de la información, se codificaron todos los datos que permitieran identificar al participante con el fin de proteger los datos personales, resguardar su identidad y asegurar su anonimato. Las investigadoras se comprometieron explícitamente a guardar la debida confidencialidad sobre los datos personales de los participantes en el proyecto, tanto en los procesos de su obtención, tratamiento y conservación, como en la posterior publicación de los resultados ${ }^{[b]}$.

\section{RESULTADOS}

\section{El cuidado y las dificultades para construir un discurso profesionalizado de la ocupación}

Tal como señalamos, la enfermería tiene la peculiaridad de definirse casi exclusivamente en términos de su misión y objetivo de brindar cuidado. De hecho, entre las personas entrevistadas, el componente cuidado de la ocupación es realzado para definir su especificidad y especialidad frente a otras profesiones de la salud, sobre todo la médica:

Tengo que estar asentada en mi rol, cuando sé cómo puedo participar, porque yo tengo bien claro que mi tarea es diferente a la del médico. El médico diagnostica, yo soy especialista en cuidado, entonces puedo hablar desde ahí. Pero tengo que estar segura, y eso me lo tiene que dar el sustento del conocimiento. (Directora de carrera, universidad pública)

Enfermera 2: -Nos pagan para brindar cuidado.

Enfermera 17: -No sos un ayudante sino parte del equipo de salud.

Enfermera 6: -Parte del equipo interdisciplinario para tomar decisiones en el tratamiento de cuidado del paciente, no solo acatar órdenes. (Entrevista grupal No.1)

No obstante, este discurso que apunta a construir el trabajo de cuidado como una especialización que requiere de saberes específicos suele aparecer matizado con otras concepciones. Así, las imágenes de género que asocian la capacidad de brindar cuidado a habilidades e inclinaciones "inherentes" a la condición femenina adquieren una fuerte presencia en las reflexiones de las enfermeras mujeres entrevistadas. En este sentido, abundan las referencias a cualidades supuestamente propias de las mujeres que las habilitarían a brindar un mejor cuidado, tales como "la intuición" (que permitiría captar mejor lo que le sucede al paciente), la tendencia "innata" a proteger y/o el entrenamiento que brinda la maternidad, entre otras.

Enfermera 1: -Está el sentido de la intuición. Una por ahí ve o percibe que algo le pasa al paciente y eso es de mujer...

Enfermera 9: -El instinto que tenemos. (Entrevista grupal No. 1)

Enfermera 34: -También bueno, tal vez la mujer tiene esa... como decirlo [...] esa capacidad de proteger, de cuidar, tal vez a nosotras nos sale naturalmente cuidar. Enfermera 35: -Ya con los hijos... (Entrevista grupal No. 2)

Aun en el caso de las entrevistadas más comprometidas con la construcción de una visión más profesionalizada de la ocupación, 
las imágenes de género asociadas al cuidado permean los discursos:

Entrevistada: -Tenemos que ser claras, no somos asistentes, no somos mucamas, ¡somos especialistas en cuidado! Somos parte de un equipo interdisciplinario de trabajo. Hay que respaldar este reclamo con más formación.

Entrevistadora: - ¿Vos dirías que las enfermeras mujeres trabajan de la misma manera que los enfermeros varones?

Entrevistada: -La enfermera mujer es como más protectora del paciente. No quiere decir que no labure el enfermero pero la enfermera mujer es más protectora en los cuidados, yo creo que por la esencia misma de ser mujer [...] tiene que ver con... conmoverte, conmoverte por la situación del paciente, la situación familiar, la situación social [...] conmoverte por todo. (Referente sindical mujer, entrevista individual)

Este tipo de asociaciones que surgen entre enfermería y cualidades "innatas" femeninas coloca a la ocupación en una situación ambigua en términos de su equiparación a un trabajo "como cualquier otro", que requiere de saberes y calificaciones específicas, que supone derechos y que habilita a reclamarlos. En este sentido, por ejemplo, la presentación del propio yo y de la ocupación en clave de sacrificio -muchas veces equiparándolos al sacrificio maternal- es una situación relativamente común entre las entrevistadas mujeres y escasamente cuestionada en términos de derechos y necesidades laborales:

Sí, a todos nos mueve la vocación, cuidar al paciente, que esté bien. A veces no doy más de las piernas [la entrevistada tiene dos empleos], horas parada o corriendo de un lado a otro, o no tuve tiempo de comer, pero los ves ahí a todos que dependen de vos y eso te da una fuerza... [...] O faltaron muchos compañeros y ves que se te viene un turno fatal... es como que te tiene que gustar esto porque es muy sacrificado... muchos [estudiantes] dejan en las prácticas, no aguantan, por eso te digo que tenés que tener vocación [...] pero la satisfacción de ver que estás ayudando, que estás cuidando al otro y que el paciente te necesita es muy fuerte, te hace fuerte. (Enfermera 37, entrevista grupal No. 2)

De manera interesante, la presencia -aunque minoritaria- de varones en la conversación grupal ayudó a cuestionar estas percepciones. En este sentido, el incremento de los varones que viene siendo observado en las cohortes más jóvenes de la ocupación ${ }^{(25,26)}$ podría constituir un elemento importante para deconstruir los estereotipos de género asociados a la ocupación:

Enfermero 10: -Perdón, pero un varón también puede cuidar. Además los varones también tienen hijos. ¿Qué, porque soy varón voy a cuidar peor?, [en referencia a la asociación entre maternidad y capacidad de cuidado].

Enfermero 8: -No, yo no estoy de acuerdo. Nos entrenamos para cuidar y no lo vamos a hacer diferente por ser mujer o varón, [en referencia al carácter instintivo y femenino del cuidado que sugiere una compañera].

Enfermera 2: -Sí, tenés razón, lo que ella dice es porque históricamente lo que tiene que ver con los cuidados... ese rol estuvo asignado a la mujer... (Entrevista grupal No. 1)

\section{La desvalorización del cuidado vista a través de prácticas y políticas institucionales}

La asociación cultural entre cuidado e imágenes estereotipadas de género sin duda presenta correlatos en la valoración social y económica de esta ocupación. En este apartado exploramos cómo se manifiesta la devaluación del cuidado en enfermería a través de prácticas y políticas institucionales.

Una primera cuestión que evidencia la desjerarquización del cuidado a nivel institucional tiene que ver con un sistema de 
salud que emplea recursos humanos en enfermería con niveles mínimos de calificación. En efecto, esta ocupación puede comenzar a ejercerse con una preparación básica: de acuerdo a los últimos datos disponibles, casi la mitad de los recursos humanos de esta ocupación (el $48 \%$ ) detenta el título de "auxiliar", que solo requiere de un año de instrucción en cuidados de baja complejidad. Cabe aclarar que, hasta 2006, se podía acceder a la formación en auxiliar de enfermería con educación primaria completa. A partir de la sanción de la Ley Nacional de Educación se comenzó a exigir la educación secundaria. El subgrupo que le sigue en términos de su peso relativo es el del personal de enfermería con formación técnica (el $41 \%$ de esta fuerza de trabajo), la cual implica tres años de formación en instituciones terciarias o universitarias. Por último, tan solo un $11 \%$ cuenta con una licenciatura en la ocupación (Figura 1). Sin duda, esta situación no se encuentra desligada de la escasez de recursos humanos, pero es importante señalar que, más allá de algunas iniciativas puntuales $y / 0$ discontinuas ${ }^{(27)}$, no ha sido acompañada de políticas extendidas y sostenidas de reconversión de los niveles

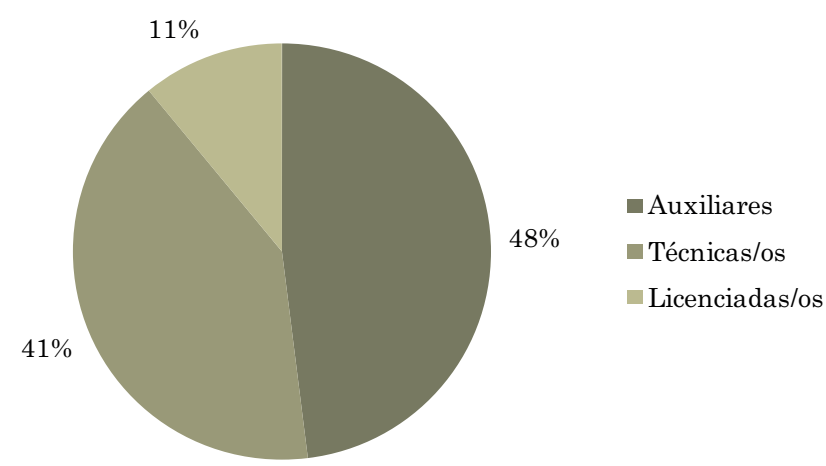

Figura 1. Distribución porcentual de la profesionalización de los recursos humanos en enfermería. Total país, Argentina, 2014.

Fuente: Red Federal de Registros de Profesionales de la Salud (REFEPS), Sistema Integrado de Información Sanitaria Argentina (SISA), Ministerio de Salud de la Nación. más bajos de calificación, ni con planes nacionales de formación del personal profesional en enfermería.

Una segunda cuestión, estrechamente vinculada con la anterior, tiene que ver con el lugar institucional en el que se ubica, o en el que es confinado, el personal de enfermería. Mientras que el personal médico forma parte de los planteles "profesionales" de las instituciones, la enfermería integra, la mayor parte de las veces, la planta de personal "no profesional" del sistema de salud. En este sentido, las enfermeras y los enfermeros forman parte de un conjunto heterogéneo de ocupaciones integrado por personal administrativo, de mantenimiento, de limpieza, camilleros, entre otros. Asimismo, y en función de esta división, en el sector público el personal de enfermería suele quedar fuera de lo que se denomina la "carrera hospitalaria", la cual presenta remuneraciones relativamente más altas que las del personal no profesional y genera oportunidades reguladas de promoción y ascenso, además de facilitar el acceso a cargos jerárquicos, en un esquema que tiende a excluir a la enfermería. Esto mismo observa en forma crítica un delegado sindical respecto a la situación del subsector público de salud en la Ciudad Autónoma de Buenos Aires:

Lo que nosotros proponemos es un escalafón sociosanitario global [...] que abarque desde los médicos hasta el personal de limpieza, a todos los que trabajamos para la salud. Porque ahora lo que tenés es un sistema jerárquico y elitista, lo que pasa ahora es que tenés a los médicos en lo que se llama la "carrera hospitalaria" que es el escalafón salarial en el que entrás si sos médico y el resto va todo al escalafón general [...] Ya desde el vamos la enfermería no se considera parte de la carrera hospitalaria, ese no es un dato menor, ya te pone en un lugar desvalorizado social y lógico, en lo económico también... (Referente sindical varón, entrevista individual)

No obstante, Ilama la atención que las enfermeras y los enfermeros entrevistados no 
suelen cuestionar espontáneamente esta segmentación ocupacional fundante del sector salud. En general, las comparaciones de las que se sirven de parámetro para evaluar que la ocupación "no es valorada" tienden a circunscribirse al salario de otras ocupaciones de la salud "no profesionales" dentro de sus establecimientos de trabajo, tales como mucamas, administrativas/os, etc.

Enfermera 20: -Que nosotros tenemos mil pesos más de básico que las mucamas, o mil quinientos. Está bien las mucamas tienen que ganar bien, me parece bárbaro.

Enfermera 17:-Pero no está reconocido, vos te preparás, estudiás...

Enfermera 23: -Yo cobro igual que los de limpieza. En serio.

Enfermera 2: $-Y$ con un administrativo tampoco hay mucha diferencia. $Y$ por ahí tienen el secundario. No es para desmerecer a nadie. Cada uno tiene su responsabilidad. Pero si vamos a comparar sueldos... conozco administrativas que tienen dos personas a cargo y gana el doble que yo. Yo tengo cinco o seis personas a cargo. (Entrevista grupal No. 1)

Esta escisión entre el personal profesional y no profesional tiene un correlato sindical que la refleja, a la vez que la reproduce. Mientras que el personal médico forma parte de asociaciones profesionales, relativamente homogéneas y con mayor poder de negociación que cualquier otro tipo de ocupación de la salud, la enfermería es representada por sindicatos mucho más heterogéneos y con menor capacidad de puja. En el sector privado, este rol lo asume la Federación de Asociaciones de Trabajadores de la Sanidad Argentina (FATSA) que nuclea al personal no médico de la salud privada. En el ámbito público, el panorama es mucho más complejo. Allí las y los trabajadores pueden estar representados por grandes sindicatos estatales, tales como la Asociación de Trabajadores del Estado (ATE) o la Unión de Personal Civil de la Nación (UPCN) ${ }^{(18)}$, y/o por sindicatos de empleados públicos provinciales y municipales. Asimismo, existen algunos sindicatos específicos de los trabajadores no profesionales de la salud pública, como el Sindicato de la Salud Pública, que reviste un peso significativo en la provincia de Buenos Aires.

A partir de este panorama, no es de extrañar el fuerte reclamo que realiza la mayoría de las enfermeras y los enfermeros entrevistados respecto de situaciones de interacción laboral en las que se evidencia la desvalorización social de la enfermería. En nuestra indagación, el personal de enfermería entrevistado reclama que el personal médico "se limita a impartir órdenes", que no existe la interconsulta con la enfermería e incluso manifestaron sentirse tratados como "asistentes" o "ayudantes". En efecto, y tal como lo que señala la bibliografía sobre el tema, el desarrollo de las tareas de la enfermería se encuentra marcado por un sistema históricamente estructurado en torno a nociones patriarcales y médico-hegemónicas ${ }^{(27)}$. Si la interacción con el personal médico sugiere importantes continuidades en este sentido, también este parece ser el caso en la interacción del personal de enfermería con los pacientes y sus familias. Es así que sus relatos indican que, en el sector privado, las enfermeras son frecuentemente confundidas $y$ tratadas como mucamas mientras que, en el sector público, los reclamos apuntan a que el personal de enfermería se encuentra entre las primeras víctimas de agresiones (verbales e incluso físicas) ante situaciones de escasez de recursos que dificultan la atención. Este tipo de experiencias, que constituyen un ángulo más para dar cuenta de la posición subordinada de la enfermería dentro del sistema de salud, no pueden ser entendidas sino a la luz de relaciones de poder que se sustentan en construcciones culturales respecto a la (des) jerarquización de las labores de cuidado ejercidas mayoritariamente por mujeres.

\section{Los desafíos adicionales que plantea la descentralización del sector salud}

Si bien el componente cuidado de la ocupación marca desafíos importantes en lo que 
atañe a la valorización social y económica de la ocupación, tal como señalamos en la introducción, existen otras variables que pueden influir sobre esta situación. En este apartado exploramos los obstáculos y desafíos adicionales que implica el desarrollo de las actividades de la enfermería en el marco de un sistema de salud altamente descentralizado y fragmentado en términos políticos, administrativos y financieros.

La escasez de datos estadísticos sobre el sector salud en nuestro país no nos permite cuantificar con precisión la distribución de la enfermería en los distintos subsectores del sistema. En vista de las limitaciones comentadas podemos aproximarnos a este fenómeno a través de algunos indicadores indirectos.

Por un lado, aunque no permite aislar a la enfermería como ocupación específica, la Encuesta Permanente de Hogares (EPH) proporciona datos sobre la distribución total de los recursos humanos en salud según sector público o privado. Tal como muestra la Figura 2, de acuerdo a los últimos datos disponibles del año 2014, el 42,3\% del personal de salud declaraba que su ocupación principal tenía lugar en la esfera pública, mientras que el 55,1\% declaró estar inserto en establecimientos privados y un $2,5 \%$ de

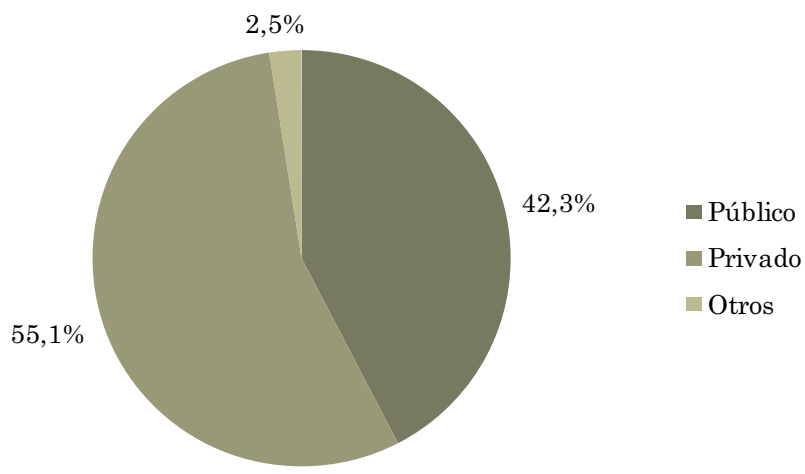

Figura 2. Distribución porcentual de ocupados en el sector salud, según subsector público/privado. Principales aglomerados urbanos, Argentina, 2014.

Fuente: Encuesta Permanente de Hogares, segundo trimestre, 2014. los encuestados declaró trabajar en "otro tipo de instituciones". Si bien este último dato podría corresponderse con el subsector de obras sociales, la falta de consignación explícita en el diseño de la encuesta no permite afirmarlo con certeza.

Por otro lado, un indicador proxy adicional para aproximarse a los diferentes tipos de inserción de los recursos humanos del sector es la distribución de establecimientos de salud por subsector. Sin duda, esta aproximación implica la lógica distorsión que acarrea el tamaño diferencial de los establecimientos $y$, por ende, la cantidad variable de recursos humanos que cada uno pueda emplear. No obstante, frente a la ausencia de fuentes de información respecto a un fenómeno tan relevante cuando se analiza al personal de salud de nuestro país, se presentan estos datos a fin de generar un primer acercamiento al tema. De acuerdo a la información del Registro Federal de Establecimientos de Salud ${ }^{(28)}$, los establecimientos dependientes del sector público representan al $45 \%$ del total (incluyendo todos los establecimientos asistenciales de los subsectores oficial, obras sociales y privado cuya actividad consista exclusivamente en el cuidado y la atención de la salud en cualquiera de sus modalidades: atención ambulatoria, internación, hospital de día, atención domiciliaria programada, diagnóstico, tratamiento, medicina preventiva, emergencias y traslados, y cualquiera de las combinaciones).

Un desglose más detallado según la jurisdicción de dependencia indica que la mayoría de estos establecimientos son provinciales (un $27 \%$ del total de establecimientos en el país), seguidos por los municipales (que aglutinan al $17 \%$ del conjunto), mientras que los nacionales representan un porcentaje ínfimo (apenas el 0,2\% del total). Por su parte, el sector privado concentra a más de la mitad de los establecimientos de salud del país (el $53 \%$ ). Por último, la tendencia de las obras sociales a subcontratar prestaciones tanto en el sector público como privado se refleja en una baja proporción (de tan solo el 1\%) de establecimientos de salud administrados en forma directa por estas entidades (Figura 3). 
Si bien los datos expuestos impiden afirmaciones precisas respecto a la distribución del personal de enfermería por sector, dejan en claro que la inserción del personal de enfermería en nuestro país -así como la de los trabajadores de la salud en general- tiene lugar en escenarios disímiles. La primera división que aparece como más visible es la de las esferas pública y privada. No obstante, como veremos a continuación, al interior de estas el panorama también presenta una fragmentación marcada. En el caso del sector público, esa fragmentación está preponderantemente signada por el nivel jurisdiccional de los establecimientos. En el sector privado, las diferencias encuentran su anclaje en un amplio espectro de empresas que difieren en el capital y la escala que manejan, el costo de sus servicios y la calidad de sus prestaciones. ¿Cómo se refleja esta heterogeneidad imperante en la esfera del trabajo de enfermeras y enfermeros de acuerdo a sus propias percepciones?

En primer lugar, surgen comparaciones generales respecto a la inserción público/ privada. Una primera cuestión que suelen mencionar las personas entrevistadas es la mayor estabilidad que garantizaría la inserción en el sector público, que implicaría un trabajo "para siempre" ("es para toda la vida", "tenés que matar a alguien para que te echen"). En este sentido, no resulta sorprendente que las personas entrevistadas relaten que el ingreso al sector público es menos permeable que el del sector privado ("cuesta muchísimo entrar", "tenés que tener la suerte de que abra una vacante").

En general, el tipo de inserción en el sector público aparece vinculado a la estabilidad y la formalidad. En efecto, la problemática de la precariedad contractual surge con más fuerza en el sector privado, especialmente en empresas pequeñas, y vinculada al trabajo no registrado. Si bien ha sido observado que en el sector público existen formas precarizadas de contratación -los regímenes de contratación sin relación de dependencia, efectivizados por medio de locaciones de servicio o bien como locaciones de obra ${ }^{(16)}$ - estas formas impactarían con más fuerza a los profesionales médicos ${ }^{(8)}$.
Depende del tipo de establecimiento [...] hay de todo [...] desde grandes grupos de excelencia a pequeños geriátricos, empresas chiquitas de cuidados domiciliarios [...] a veces con condiciones laborales terroríficas [...] A veces viene gente en negro y les digo: "pero usted está en negro, lo primero que tenemos que hacer es regularizar su situación". Y ellos: "no, no el patrón, no me puede poner" [en blanco] y dicen: "no, no, no" y se van. (Referente sindical mujer, entrevista individual).

Otro de los temas problemáticos que remarcan frecuentemente las personas entrevistadas es la sobrecarga de pacientes y la consiguiente necesidad de "hacer un millón de cosas", "estar en todo". Si bien el tema atraviesa tanto al sector público como al privado, los relatos sugieren que la situación presenta heterogeneidades al interior de este último ("depende de donde trabajes").

En efecto, dentro de la esfera privada surge una clara distinción entre la "categoría" de los establecimientos. En palabras de una referente sindical de este sector:

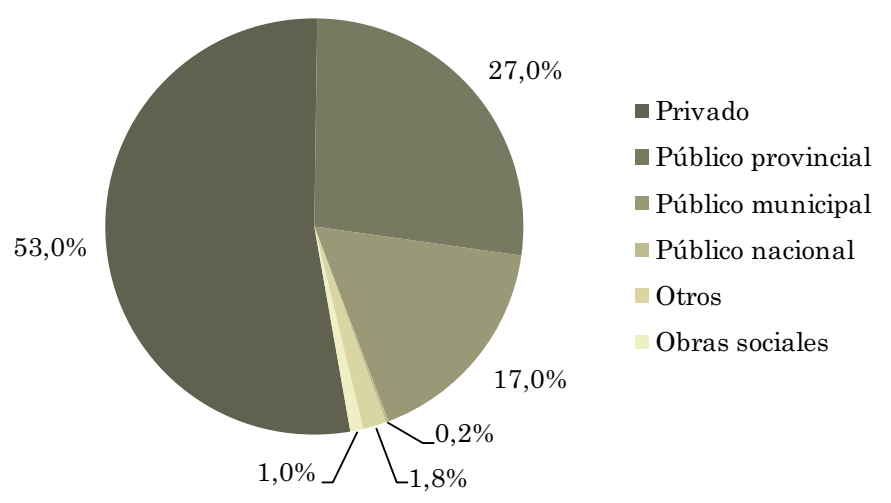

Figura 3. Distribución porcentual de establecimientos de salud, según subsector y jurisdicción. Total país, Argentina, 2014.

Fuente: Registro Federal de Establecimientos de Salud (REFES), Ministerio de Salud de la Nación. 
Hay lugares en los que se trabaja muy bien, con condiciones... no te voy a decir óptimas porque no existe lo óptimo pero con muy buenas condiciones laborales y otras que es terrible, con mucha sobrecarga de pacientes. Lo que más prima es la sobrecarga de pacientes [...] en las clínicas que te venden el servicio, ahí tenés una muy buena relación médico-paciente [...] obviamente tienen un cliente, que ellos consideran sumamente exigente, entonces tratan de no sobrecargar a la enfermera [...] después tenés un degradé [...] hay clínicas te diría de medio pelo. Mutualidades que tienen convenios con obras sociales, dependientes del PAMI [Instituto Nacional de Servicios Sociales para Jubilados y Pensionados]... Ios geriátricos en general [gesto de desaprobación]... los dueños, digo, son patrones de estancia, entonces se trabaja en muy malas condiciones. (Referente sindical mujer, entrevista individual)

En lo relativo a remuneraciones -siempre en el marco de lo que se señala como "salarios bajísimos"-, al momento de la entrevista las personas coincidieron en señalar que, con excepción de los establecimientos públicos de la Ciudad Autónoma de Buenos Aires, las remuneraciones del sector privado eran superiores a las del público. No obstante, las diferencias salariales entre uno y otro sector se presentan como una situación coyuntural, sujeta a vaivenes presupuestarios:

Hubo durante mucho tiempo migración del privado al público, te hablo de unos cuatro o cinco años atrás, cuando el sector público abrió sus puertas para cubrir vacantes, en ese momento los salarios [del sector público] eran mejores que los del privado [...] ahora los salarios del sector privado están más altos que el público, bastante más altos te diría. (Referente sindical mujer, entrevista individual)

Asimismo, dentro del sector público, las comparaciones que realizaron las y los entrevistados entre salarios de distintas jurisdicciones reflejan lo heterogéneo de la situación. Más allá de la dispersión de los relatos, la situación subyacente parece ser la esperable: un escenario donde las jurisdicciones con mayor presupuesto pueden ofrecer remuneraciones algo mejores.

Yo del [hospital] municipal cuando pude me fui. Pasé al provincial, porque paga mejor. $Y$ eso que el municipal me quedaba re cerca de casa, pero igual no conviene. Todo el mundo se quiere pasar [del nivel municipal al provincial]. (Enfermera 32, entrevista grupal $N^{\circ} 2$ )

Acá todos aspiran a entrar al nivel provincial [se refiere a la provincia de Buenos Aires]. Es que el sueldo de los municipales es bajísimo, mucho más bajo que el provincial. Te puedo nombrar casos concretos, en 25 de Mayo, Coronel Suárez... los sueldos básicos son vergonzosos. Ahora, si te vas al Gobierno de la Ciudad [de Buenos Aires] los sueldos están muy por encima del provincial. (Coordinador de carrera, universidad pública, entrevista individual)

Nosotros por ahí vemos cómo la gente va adquiriendo experiencia, se van perfeccionando, algunos inclusive estudian, tratamos de que estudien, pero por ahí cuando ya están formados consiguen un puesto en Ciudad [se refiere a la Ciudad de Buenos Aires] y se van. (Directora de departamento de Enfermería, hospital municipal, entrevista individual)

Por último, y completando el panorama de dispersión salarial, las y los trabajadores de diferentes empresas del sector privado - dependiendo de su envergadura- pueden negociar salarios por sobre el mínimo acordado por el sector.

Después, por ejemplo, vos decís [menciona una empresa de medicina prepaga] es una empresa que gana mucho más y se consiguen extra-paritarias por encima del básico firmado en convenio. 
En [menciona otras tres empresas de medicina prepaga] pasa lo mismo... los negocia el sindicato con los delegados del establecimiento y la patronal específica del caso... tienen buenos sueldos, dentro de todo, ¿no? (Referente sindical mujer, entrevista individual)

La situación de fragmentación en términos de experiencias laborales y salariales tiene como lógico correlato una estructura sindical de la ocupación altamente escindida. Esta escisión puede representarse en dos planos. Por un lado, la fragmentación sindical refleja y reproduce la estructura política del sector salud, que diferencia niveles de representación a nivel privado y público $\mathrm{y}$, dentro del público, se ramifica en numerosas entidades que negocian con cada jurisdicción. Por otro lado, como mencionamos en el apartado anterior, la dispersión sindical también tiene que ver con la jerarquización del personal de la salud entre "profesionales" y "no profesionales", quedando la enfermería mayoritariamente encasillada en esta última categoría (Figura 4).

La importante dispersión de la representación gremial de la ocupación resta, sin duda, capacidad de negociación a estas/ os trabajadoras/es. Si esta situación ayuda a comprender el predominio de remuneraciones bajas, también permite poner en contexto problemas como el de la extensión sistemática de las jornadas laborales (a través de la incorporación de horas extras), así como el del pluriempleo generalizado en la ocupación.

En efecto, uno de los mecanismos "compensadores" de salarios bajos lo constituye la extensión de las jornadas laborales en un mismo establecimiento a través de las horas extras. Si bien se trata de una práctica generalizada, existen algunos matices. Un caso extremo lo constituye el de la Ciudad de Buenos Aires, donde el personal de enfermería puede Ilegar a duplicar la cantidad de horas de la jornada laboral a través de los llamados "módulos", que son el equivalente a las horas extras y se pautan en forma de bloques horarios de seis horas cada uno.
Según las aserciones de las personas entrevistadas, este componente precarizado de la remuneración a través de las horas extras sistemáticas (no es estable, genera jornadas interminables) se encuentra naturalizado entre quienes desempeñan la ocupación:

Está tan instalado el tema del módulo [...] es como una legitimación de un doble sueldo. [...] los módulos se reparten [...] es un recurso de poder. (Funcionario del área de recursos humanos, Ministerio de Salud, entrevista individual)

Mientras que en el sector privado la modalidad de las horas extras también se encuentra ampliamente extendida, el caso de los trabajadores hospitalarios dependientes de la provincia de Buenos Aires ha experimentado un cambio significativo que ha despertado expectativas de réplica entre muchos trabajadores de otros subsectores y jurisdicciones. En este ámbito las tareas han sido recientemente declaradas como "insalubres". Esta decisión ha implicado, entre otras cuestiones, la reducción de la jornada laboral de ocho a seis horas diarias, la posibilidad de jubilación anticipada, así como la restricción de la cantidad de horas extras que se pueden trabajar ${ }^{[c]}$.

Independientemente de las distintas regulaciones en torno a la cantidad y la forma en que pueden agregarse horas adicionales a la jornada laboral en un mismo establecimiento, existe otra modalidad que asume la sobreexplotación sistemática de esta fuerza de trabajo. Nos referimos a la situación generalizada de pluriempleo, problemática que ya ha sido observada en trabajos sobre el sector salud en general $(22,29,30)$ y al que hacen referencia de manera reiterada las personas entrevistadas para el caso de la enfermería en particular:

La mayoría de los enfermeros del país tienen doble empleo, es muy raro que encuentres un enfermero con un solo empleo [...] están los que trabajan de lunes a viernes y después sábados, domingos y feriados, se Ilaman "franqueros" [es 


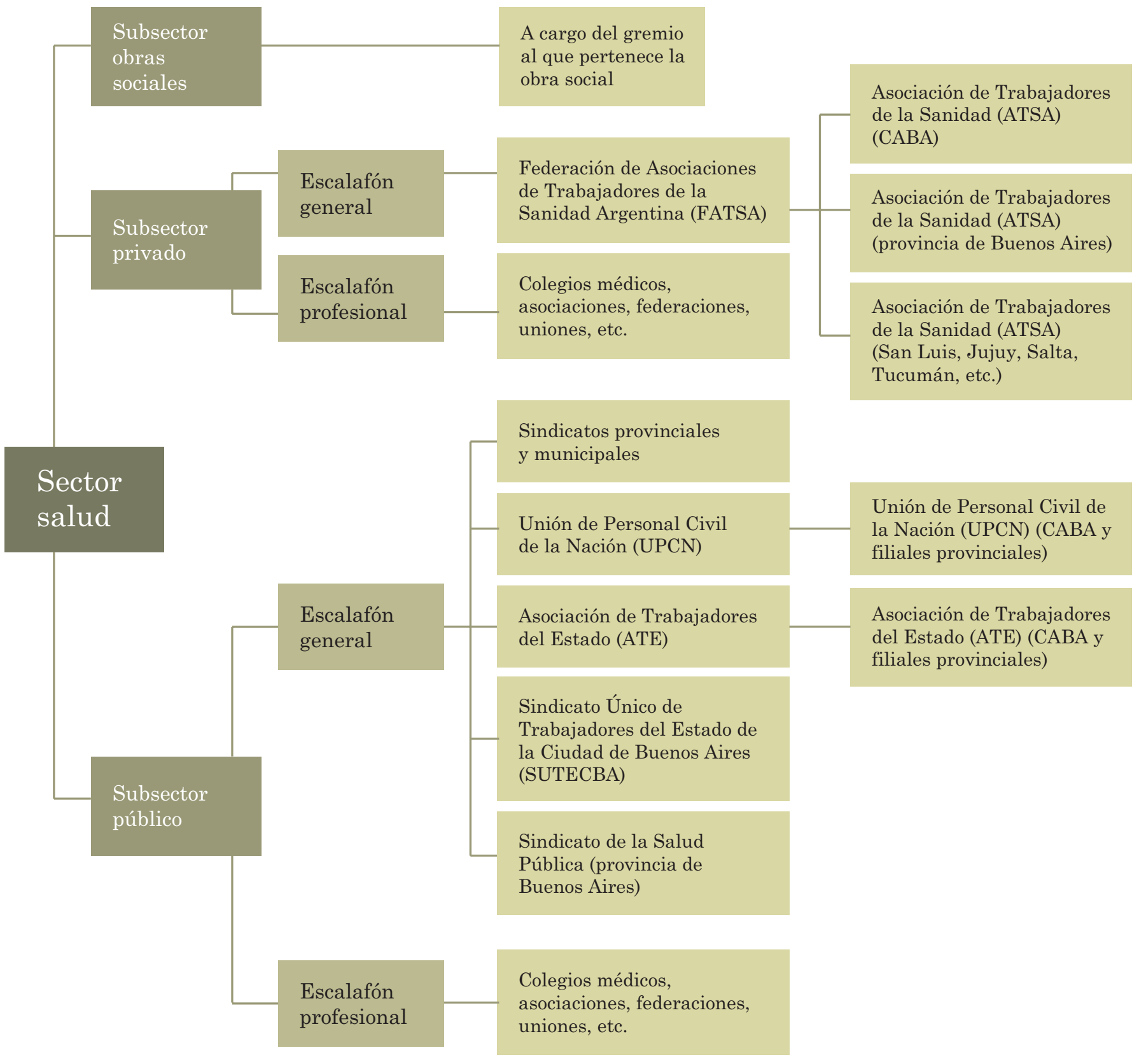

Figura 4. Representación gremial en el sector salud. Argentina, 2014.

Fuente: Elaboración propia. 
decir, el segundo empleo se realiza en los "francos" o días libres del primero] y los que trabajan de noche tienen que trabajar noche por medio. Pero vos podés tener un enfermero que trabaja noche por medio en una institución y sale de esa guardia y va a trabajar a otro hospital a la mañana. Después vos me decís ¿cómo queda ese enfermero, cómo puede cuidar ese enfermero? (Directora de carrera de Enfermería, universidad privada, entrevista individual)

Aquí, la fragmentación del sector salud opera incluso facilitando esta situación, puesto que el desempeño simultáneo en diferentes subsectores o jurisdicciones disminuye potenciales incompatibilidades a la vez que habilita y legitima el trabajo a destajo. Tal como señala, a modo de ejemplo, un funcionario del Ministerio de Salud:

El sistema está preparado para el pluriempleo, es así, y el doble empleo existe en todos lados y hasta es estructural... [Un patrón común es el de] la enfermera que tiene por un lado un laburo que es el laburo estable, que en general es en el sector público, con todos los beneficios que te da el sector público respecto de licencias, y un empleo que es en el sector privado [...] en donde ellas manejan los francos y las ausencias que usan en el otro. Los francos y las ausencias que las pueden usar a veces para trabajar en otro lado y otras veces los usan porque están destruidas. (Funcionario del área de recursos humanos, Ministerio de Salud, entrevista individual)

\section{DISCUSIÓN Y CONCLUSIONES}

A lo largo de este artículo hemos buscado exponer de qué manera la desvalorización social del cuidado -con todas sus connotaciones de género- puede ser interpretada como un factor de suma importancia a la hora de explicar las condiciones laborales de la enfermería. En efecto, el componente cuidado de la ocupación se encuentra estrechamente asociado a estereotipos tradicionales de género que operan asociando los saberes específicos de la enfermería con saberes y habilidades supuestamente "inherentes" a la condición femenina. La alta permeabilidad de este tipo de discursos, presente incluso en los relatos de quienes ejercen la profesión, sugiere que es necesario un arduo trabajo político-institucional para deconstruir este tipo de visiones que desjerarquizan el tipo de trabajo realizado. Asimismo, a nivel institucional, la devaluación social del cuidado que ejerce la enfermería se expresa, por ejemplo, a través de los escasos niveles formales de calificación que requiere el sistema de salud para estos recursos humanos, del confinamiento de la ocupación a las grillas y regímenes laborales de los trabajadores "no profesionales" del sector salud o de su posición subordinada frente al personal médico en las interacciones laborales cotidianas, entre algunos de los indicadores observados.

Por otro lado, también se ha explorado cómo otros factores -indagamos aquí sobre la fragmentación del sector salud- operan, en este caso, reforzando la "penalización" laboral del cuidado. Si bien la fragmentación del sistema afecta indudablemente al conjunto de las ocupaciones de la salud, son aquellas que se encuentran en una situación políticamente más vulnerable -como el caso de la enfermería- las que resultan relativamente más afectadas. Así, en el marco de condiciones de trabajo vulnerables, la descentralización del sistema marca diferentes experiencias laborales según el tipo de inserción, situación que incide en la posibilidad de articular reclamos. Esta posibilidad también se ve afectada por la significativa dispersión sindical de este colectivo laboral: a la escisión gremial de la enfermería respecto a las ocupaciones médicas, se le suma la multiplicación de las representaciones sindicales según el subsector de inserción, así como también las diferentes jurisdicciones de pertenencia dentro del subsector público.

En efecto, el tema de la falta de poder político de este colectivo laboral -ya sea 
por el encasillamiento de sus labores dentro de estereotipos tradicionales de género, por su desempeño laboral en el marco de un sistema de salud médico-hegemónico, o por su dispersión en función de un sistema de salud fragmentado- se presenta así como un eje crucial a abordar en pos de mejorar las condiciones laborales de la ocupación.

Justamente a partir de este posicionamiento vulnerable, las intervenciones gubernamentales revisten un rol fundamental para mejorar las condiciones laborales de la ocupación. En este sentido, consideramos pertinente concluir este artículo revisando algunos ejes de un conjunto de iniciativas legislativas sobre la enfermería que han surgido desde el poder político en los últimos años. Se trata de una serie de proyectos de ley que suelen abordar temas relativos a muchas de las problemáticas claves de la ocupación que se han señalado a lo largo de este trabajo.

Por un lado, la mayoría de estos proyectos propicia la declaración de la emergencia nacional de los recursos humanos de enfermería, proponiendo distintas versiones para la implementación de un plan nacional de formación para la ocupación ${ }^{(31,32,33,34,35,36,37)}$. Este tipo de políticas apuntaría no solo a aliviar el déficit cuali-cuantitativo de recursos humanos sino también a empoderar al colectivo enfermero dotándolo de credenciales educativas que respalden el valor de sus saberes específicos. En este sentido, existen experiencias internacionales interesantes que ponen en evidencia diferentes posibilidades de avance en la materia. Brasil, por ejemplo, es un país que en el marco de una situación crítica en materia de calificación del personal de enfermería puso en marcha un plan nacional de jerarquización de la ocupación a través del Proyecto de Formación de los Trabajadores del Área de Enfermería (PROFAE), implementado a partir del año 2000 con financiamiento del Banco Interamericano de Desarrollo y del Tesoro Nacional. Este proyecto encaró, desde el Ministerio de Salud, un plan nacional de capacitación del personal de enfermería en ejercicio para todos los estados del país. La intervención devino en un proceso masivo de reconversión del personal en los estratos de menor calificación, fundamentalmente del nivel empírico al de auxiliar y del auxiliar al técnico ${ }^{(16,38)}$. El caso europeo provee un ejemplo interesante con relación al fomento de la formación universitaria en la ocupación. A partir de la creación del Espacio Europeo de Educación Superior (EEES) se buscó armonizar los distintos sistemas educativos universitarios de la Unión Europea con el fin de facilitar la movilidad profesional, estudiantil y la cooperación científica en general. La enfermería, en particular, experimentó cambios importantes, ya que la adhesión al EEES fomentó la elevación de los requerimientos de credenciales educativas para participar de las oportunidades que provee el espacio. En este sentido, el EEES promovió la expansión del dictado de la carrera en los espacios universitarios, generando una lenta pero progresiva tendencia a la licenciatura universitaria para ejercer la ocupación, así como también los estudios de posgrado en la disciplina ${ }^{(15)}$. La valorización de la ocupación a través de su jerarquización en el ámbito universitario no constituye un tema menor, en tanto la posiciona en un lugar más atractivo como elección de carrera, en un contexto internacional en el que la escasez de recursos humanos en enfermería -y particularmente de personal calificado- constituye una problemática recurrente.

Por otro lado, los proyectos de ley nacionales en danza suelen coincidir también en torno a la necesidad de generar y declarar insalubre la actividad de enfermería en todas las áreas en las que se desarrolle con el fin de establecer una serie de licencias especiales, un régimen previsional diferencial anticipado, así como un máximo de jornada laboral $^{(39,40,41,42)}$. Este punto también aparece como central en función de las problemáticas planteadas por las personas entrevistadas, sobre todo en relación con las extendidas situaciones de estrés laboral. No obstante, tal como lo evidencia la situación del personal de enfermería de la provincia de Buenos Aires -donde este tipo de política se está implementando en forma paulatina-, si estas medidas no se acompañan con aumentos salariales, el objetivo de aliviar la carga laboral 
de la ocupación puede desdibujarse. En este sentido, una baja de la carga horaria en el marco de bajos ingresos perpetúa e incluso puede intensificar las situaciones de pluriempleo existentes.

El punto anterior nos lleva a un tercer y último eje que nos interesa rescatar, que consiste en la propuesta de establecer remuneraciones mínimas mucho más altas que las actuales para la ocupación (medidas en términos de una determinada cantidad de salarios mínimos vitales y móviles) ${ }^{(41)}$. Un aspecto interesante de esta propuesta es su carácter nacional, aspecto que plantearía una posible solución al tema de la profunda fragmentación salarial de la ocupación, así como también el hecho de que premia fuertemente en términos salariales a los niveles más altos de capacitación, constituyéndose así en un incentivo a la profesionalización de la ocupación.

Resulta claro que cualquiera de estas propuestas (que, desde ya, no pretenden agotar el espectro de iniciativas posibles dirigidas a la ocupación) no implican un desafío meramente técnico sino también, y fundamentalmente, político. Se trata de proyectos que implican cuestionar formas establecidas y arraigadas de distribución de recursos (materiales, pero también simbólicos) y, por ende, relaciones de poder. Así, la posibilidad de implementar cualquiera de estas (u otras) propuestas depende fuertemente, por un lado, de la conciencia, la voluntad y las estrategias que puedan surgir en los ámbitos de decisión gubernamentales. Y, por otro lado, un escenario ideal incluiría una fuerte incidencia del propio colectivo enfermero en generar iniciativas y presión política. La introducción de demandas propias en la agenda pública por parte de los actores sociales involucrados resulta clave, e implica la necesidad de construcción de poder político por parte del colectivo enfermero con el fin de impulsar estas iniciativas en pos de sus intereses ${ }^{(43)}$. En este sentido, los logros específicos que puedan generarse en materia de elevar el nivel de formación de estas/os trabajadoras/es a mediano y largo plazo constituyen un factor estratégico. La nivelación "hacia arriba" de la formación de las enfermeras y los enfermeros generaría elementos y argumentos para reclamar un lugar en las estructuras profesionales del sector salud así como también para estimular mayores niveles de articulación entre sus organizaciones, contribuyendo de esta manera a construir un mejor posicionamiento político (y por ende, laboral) de la ocupación.

\section{AGRADECIMIENTOS}

El presente artículo se enmarca dentro del proyecto de investigación "Las condiciones laborales de las y los trabajadores del cuidado. Procesos sociales y políticas públicas detrás de su configuración", con sede en la Universidad Nacional de General Sarmiento y financiado por la Agencia Nacional de Promoción Científica y Tecnológica Argentina (PICT 2011-0622).

\section{REFERENCIAS BIBLIOGRÁFICAS}

1. Folbre N. Demanding Quality: Worker/consumer coalitions and "high road" strategies in the care sector. Politics and Society. 2006;34(1):11-32.

2. Nelson J. Of markets and martyrs: Is it ok to pay well for care? Feminist Economics. 1999;5(3):43-55.

3. Esquivel V. La economía del cuidado en América Latina: Poniendo a los cuidados en el centro de la agenda. El Salvador: PNUD; 2011. 
4. Vega C, Gutiérrez-Rodríguez E. Nuevas aproximaciones a la organización social del cuidado: Debates latinoamericanos. Íconos. 2014;18(3):9-26.

5. Benería L. Reproduction, production and the sexual division of labour. Cambridge Journal of Economics. 1979;3(3):203-225.

6. Jelin E. Familia y unidad doméstica: Mundo público y vida privada. Buenos Aires: CEDES; 1979.

7. Hochschild AR. The second shift: Working parents and the revolution at home, (with Anne Machung). New York: Viking Penguin; 1989.

8. England P, Budig M, Folbre N. Wages of virtue: The relative pay of care work. Social Problems. 2002;49:455-473.

9. Razavi S, Staab S. Underpaid and overworked: A cross-national perspective on care workers. International Labour Review. 2010;149(4):407-422.

10. Budig, MJ, Misra J. How care-work employment shapes earnings in cross-national perspectives. International Labour Review. 2010;149(4):441-460.

11. Esquivel V. Care workers in Argentina: At the crossroads of labour market institutions and care services. International Labour Review. 2010;149(4):477-496.

12. Programa Naciones Unidas para el Desarrollo (PNUD). El sistema de salud argentino y su trayectoria de largo plazo: logros alcanzados y desafíos futuros. Buenos Aires: Programa Naciones Unidas para el Desarrollo; 2011.

13. Tobar F, Olaviaga S y Solano R. Complejidad y fragmentación: las mayores enfermedades del sistema sanitario argentino. Documento de Políticas Públicas. Análisis $N^{\circ}$ 108. Buenos Aires: CIPPEC; 2012.

14. Zabalegui-Yárnoz A. El rol profesional en enfermería. Aquichán. 2003;3(3):16-20.

15. Organización Mundial de la Salud. Nursing and midwifery progress report 2008-2012. Ginebra: OMS; 2013.

16. Organización Panamericana de la Salud. Estudio comparativo de las condiciones de trabajo y salud de los trabajadores de la salud en: Argentina, Brasil, Costa Rica y Perú. Washington DC: OPS; 2012.

17. Aspiazu E, Baldi L, Lanari ME. Prestadores, prestatarios y pacientes: un análisis de las voces y silencios de los reclamos de los profesionales de la salud [Internet]. X Congreso Nacional de Es- tudios del Trabajo; 3-5 ago 2011; Buenos Aires, Argentina [citado 10 jul 2015]. Disponible en: http://goo.gl/MZB5ds.

18. Aspiazu E. Los conflictos laborales en la salud pública en Argentina. En: Trabajo, ocupación y empleo: Una mirada a sectores económicos desde las relaciones laborales y la innovación. Buenos Aires: Ministerio de Trabajo Empleo y Seguridad Social; 2010.

19. Lanari ME. Trabajo decente: un aporte metodológico para su estimación. Aplicación en la determinación del actual déficit de trabajo decente entre los profesionales de la salud del sector público y privado de Mar del Plata. [Tesis doctoral]. Buenos Aires: FLACSO; 2006.

20. Organización Panamericana de la Salud (OPS). Panorama de la fuerza de trabajo en enfermería en América Latina. Washington DC: OPS; 2005.

21. Organización Panamericana de la Salud, Ministerio de Salud de la Nación. Bases para un plan de desarrollo de recursos humanos en salud: Informe final. Buenos Aires: OPS, MSAL; 2005.

22. Novick M, Galín P. Flexibilidad del mercado de trabajo y precarización del empleo: El caso del sector salud. En: Organización Panamericana de la Salud. Observatorio de RRHH en Salud en Argentina: Información estratégica para la toma de decisiones. Buenos Aires: OPS; 2003.

23. Wainerman C, Geldstein R. Condiciones de vida y trabajo de las enfermeras en Argentina. Buenos Aires: CENEP; 1990. (Serie Cuadernos del CENEP No 44).

24. Maceira D, Cejas C. Recursos humanos en salud: Una agenda para el gobierno nacional [Internet]. Buenos Aires: CIPPEC; 2010 [citado 10 jul 2015]. Disponible en: http://goo.gl/YcaJAh.

25. Abramzón M. Argentina: recursos humanos en salud en 2004. Buenos Aires: OPS; 2005.

26. Róvere M. Los nuevos y renovados debates en torno de los recursos humanos en salud. En: Rico MN, Marco F, coordinadores. Mujer y empleo: La reforma de la salud y la salud de la reforma en la Argentina. Buenos Aires: Siglo XXI Editores; 2006.

27. Biernat C, Cerdá JM, Ramacciotti KI, (dir). La salud pública y la enfermería en la Argentina. Buenos Aires: Universidad Nacional de Quilmes; 2015.

28. Ministerio de Salud, Sistema Integrado de Información Sanitaria Argentina. Registro Federal 
de Establecimientos de Salud [Internet]. Buenos Aires: SIISA [citado 10 jul 2015]. Disponible en: https://goo.gl/oYKye8.

29. Organización Panamericana de la Salud. La salud de los trabajadores de la salud: Trabajo, empleo, organización y vida institucional en hospitales públicos del aglomerado Gran Buenos Aires, 2010-2012. Buenos Aires: OPS; 2013.

30. Pautassi L. El empleo en salud en la Argentina: La sinergia entre calidad del empleo y calidad de la atención. En: Rico MN, Marco F, (coord). Mujer y empleo: La reforma de la salud y la salud de la reforma en la Argentina. Buenos Aires: Siglo XXI Editores; 2006.

31. Ocaña MG, Bertol PM, Pucheta R, Garrido M. Declarar la emergencia nacional de los recursos humanos de enfermería; promover su inclusión en el artículo 43 de la ley 24.521 de educación superior y modificación de la ley 24.004 [Internet]. Honorable Cámara de Diputados de la Nación: Expediente 3208-D-2012 [citado 10 jul 2015]. Disponible en: http://goo.gl/Fuxhd3.

32. Regazzoli MC, Gardella PS, Torfe ML, Portela AA, Storani ML, Chieno MEP. Plan nacional de desarrollo de la enfermería. Declarar la emergencia nacional de los recursos humanos en enfermería. Promover su inclusión en el artículo 43 de la ley 24.521 de educación superior [Internet]. Honorable Cámara de Diputados de la Nación: Expediente 1566-D-2011 [citado 10 jul 2015]. Disponible en: http://goo.gl/ydOEJH.

33. Parrilli NMA, Rojkes de Alperovich BL, Corregido EM, Pichetto MA, Fellner LB, Corpacci LB. Proyecto de ley declarando la emergencia nacional de los recursos humanos en enfermería [Internet]. Honorable Senado de la Nación Argentina: Expediente 888/11 [citado 15 jul 2015]. Disponible en: http://goo.gl/5E0APm.

34. Osuna BI, Rojkes de Alperovich BL. Proyecto de ley declarando la emergencia nacional de los recursos humanos en enfermería [Internet]. Honorable Senado de la Nación Argentina: Expediente 1032/11 [citado 10 jul 2015]. Disponible en: http://goo.gl/mCwkZh.

35. Díaz MR, Martínez JC. Proyecto de ley declarando la emergencia nacional del personal en enfermería y estableciendo una política de Estado para su superación [Internet]. Honorable Senado de la Nación Argentina: Expediente 1041/11 [citado 15 jul 2015]. Disponible en: http://goo.gl/ GgQUaU.

36. De Narvaez F, Atanasof AN, Ferrari GAH, Gambaro N. Creación del plan federal de for- mación y empleo de enfermeros [Internet]. Honorable Cámara de Diputados de la Nación: Expediente 2558-D-2012 [citado 16 jul 2015]. Disponible en: http://goo.gl/Lc0KSk.

37. Giménez SD. Proyecto de ley creando el plan nacional de desarrollo de la enfermería [Internet]. Honorable Senado de la Nación Argentina: Expediente 1894/12 [citado 16 jul 2015]. Disponible en: http://goo.gl/83Btj6.

38. Göttems L, Alves E, Sena R. La enfermería brasileña y la profesionalización de nivel técnico: un análisis en retrospectiva. Revista Latinoamericana de Enfermagem. 2007;15(5):1033-1040.

39. Harispe G. Licencia especial paliativa preventiva del estrés laboral de 10 días hábiles a trabajadores no profesionales y trabajadores del área de enfermería del sector estatal de salud mental [Internet]. Honorable Cámara de Diputados de la Nación: Expediente 5486-D-2012 [citado 15 jul 2015]. Disponible en: http://goo.gl/i1hy4U.

40. Regazzoli MC, García-Larraburu SM. Régimen previsional especial para los trabajadores que ejercen la enfermería: modificación de la ley 24.004 [Internet]. Honorable Cámara de Diputados de la Nación: Expediente 2020-D-2012 [citado 15 jul 2015]. Disponible en: http://goo.gl/ $\mathrm{R} 754 \mathrm{Fb}$.

41. Rivas J, Gallardo MG, Puiggros AV, Chieno MEP, Donkin CG. Ejercicio de la enfermería. Régimen para el ejercicio de la enfermería. Reforma de la ley 24.004 [Internet]. Honorable Cámara de Diputados de la Nación: Expediente 7323-D-2012 [citado 15 jul 2015]. Disponible en: http://goo. gl/7LngS5.

42. Ramal M. Proyecto de ley: Trabajo insalubre y 6 horas para enfermería [Internet]. 2014 [citado 15 jul 2015]. Disponible en: http://goo.gl/fnddf4.

43. Testa M. Estrategia, coherencia y poder en las propuestas de salud (segunda parte). Cuadernos Médico Sociales. 1987;(39):3-28.

\section{NOTAS FINALES}

[a] El Área Metropolitana de Buenos Aires (AMBA) está compuesta por localidades y barrios de la Ciudad Autónoma de Buenos Aires y de su conurbación sobre la provincia de Buenos Aires (conurbano), caracterizándose por ser la concentración urbana de mayor densidad del país (aglutina alrededor del 30\% de la población total). En su territorio se ubican grandes conglomerados urbanos, donde conviven los sectores más ricos 
del país con poblaciones en condiciones de pobreza estructural. Las desigualdades presentan un patrón en términos de distribución territorial ya que los sectores de mayores ingresos tienden a concentrarse en la Ciudad Autónoma de Buenos Aires, mientras que en el conurbano predominan los sectores de ingreso bajo y medio-bajo. La excepción la constituye, por un lado, el corredor norte del conurbano -que nuclea a los sectores acomodados que residen en los suburbios de la Ciudad Autónoma de Buenos Aires- y, por otro lado, la multiplicidad de urbanizaciones privadas, aisladas y dispersas, que en las últimas décadas han comenzado a proliferar a lo largo de todo este territorio. Como correlato de este patrón desigual de distribución territorial de la riqueza, el AMBA encierra grandes diferenciales entre territorios en cobertura, complejidad y calidad de la oferta de servicios de salud, en algunos casos superiores a los que se observan entre las provincias ${ }^{(12)}$. En este contexto, la Ciudad Autónoma de Buenos Aires se diferencia particularmente del conurbano, ya que concentra la mayor parte de la estructura de servicios y de recursos humanos en salud del $\mathrm{AMBA}^{(13)}$ [b] La Resolución 1480/2011 del Ministerio de Salud indica que las investigaciones en este campo requieren, además del consentimiento informado de los participantes, de la evaluación inicial del proyecto por un Comité de Ética en Investigación. No obstante, el proyecto en el que se enmarca este trabajo no fue sometido a tal procedimiento dado que no fue solicitado por la Agencia Nacional de Promoción Científica y Tecnológica, entidad que aprobó y financió la investigación.

[c] A partir del 2013 rige un régimen de insalubridad que estableció el pase de 48 horas semanales de trabajo a 36 horas sin pérdida de ingreso (modificación del Art. 26, de la Ley 10430). En el año 2014 se agrega la posibilidad de jubilación anticipada a los 50 años de edad con 25 años de servicio. No obstante, la aplicación de este último beneficio no es automática sino que, a partir del 2014 se plantea la baja progresiva de la edad jubilatoria y los aportes exigidos hasta 2018, año desde el cual regirá de forma plena la nueva normativa.

\section{FORMA DE CITAR}

Pereyra F, Micha A. La configuración de las condiciones laborales de la enfermería en el Área Metropolitana de Buenos Aires: un análisis en el cruce del orden de género y la organización del sistema de salud. Salud Colectiva. 2016;12(2):221-238. doi: 10.18294/sc.2016.730.

Recibido: 14 de septiembre de 2015 | Versión final: 1 de febrero de 2016 | Aprobado: 10 de marzo de 2016

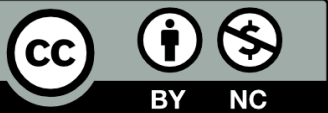

Este obra está bajo una licencia de Creative Commons Reconocimiento-NoComercial 4.0 Internacional. Reconocimiento - Permite copiar, distribuir y comunicar públicamente la obra. A cambio, se debe reconocer y citar al autor original. No Comercial - Esta obra no puede ser utilizada con finalidades comerciales, a menos que se obtenga el permiso.

http://dx.doi.org/10.18294/sc.2016.730 